\title{
Performance Analysis of Phased Array and Frequency Diverse Array Radar Ambiguity Functions
}

Shaddrack Yaw Nusend*

Koforidua Technical University, Electrical / Electronic Department, Faculty of Engineering, Ghana.

\begin{tabular}{l} 
A R T I C L E I N F O \\
\hline Article history: \\
Received: 04 April, 2017 \\
Accepted: 12 May, 2017 \\
Online: 22 May, 2017 \\
\hline Keywords: \\
Frequency diverse array \\
Phased array radar \\
Radar ambiguity functions \\
ECDF
\end{tabular}

\section{Introduction}

Phased array radar system has been widely utilized in many modern communications, radar and navigation systems [1, 2, 3]. In a standard phased-array antenna, the elements are fed coherently and phase shifters or time-delayers are used to scan the beam to the desired direction in space [4]. The offered directional gain is beneficial for target detection and tracking weak targets in the antenna looking direction and the same time suppressing strong sidelobe interferences from other directions. The phased-array antenna beam steering is fixed in an angle for all ranges and thus, the range and angle of targets cannot be directly estimated from its beamforming output due to an inherent range ambiguity [5].

Recently, a flexible array, namely, frequency diverse array (FDA) has been proposed [6, 7, 8]. The most significant FDA difference from its counterpart phased-array is that a small frequency increment, compared to the carrier frequency, is applied between the FDA elements. This frequency increment yields a range-angle-dependent beampattern for which the beam focus direction will be a function of the angle, range and time [9, 10, 11]. FDA has sparked much investigations because of its promising applications. In [12, 13, 14] FDA have been investigated in terms of range-dependent beam with applications in suppressing range ambiguous clutter. FDA for synthetic aperture radar (SAR) high-resolution imaging was reported in [15]. Furthermore, [16, 17] exploited FDA capabilities in range and angle localization of targets.
It is well established that the radar ambiguity function is a useful tool for evaluating performance metrics of radar system, such as time delay (range) and Doppler frequency resolutions and the probabilities of detection and false alarm. Moreover, it helps in selecting appropriate waveforms depending on the required performance parameters.

Therefore, in this perspective, this paper derived and analyzed the performance characteristics of phased array and FDA radar ambiguity functions in time delay and Doppler frequency resolutions. Ambiguity function performance metric is also provided in terms of empirical cumulative distribution function (ECDF). The performance characteristics are compared and FDA radar exhibited better performance results than the phased array radar.

The remaining sections are organized as follows: In Section II, conventional ambiguity function is reviewed. Section III, ambiguity functions for FDA and phased array is derived. Section IV, provides simulation results. Finally, Section V, concludes the paper.

\section{Review Of The Conventional Am- biguity Function}

In a conventional radar system, the radar ambiguity function (AF) is a common tool for investigating radar signals [18, 19, 20, 21]. The AF is a two dimensional function that describes the output of a matched filter when the input signal of time delay $t_{d}$ and Doppler frequency $f_{d}$ relative to

* Shaddrack Yaw Nusenu, Koforidua Technical University, Ghana, Email: nusenu2012gh@yahoo.com 
nominal values for which the matched filter has been designed. The AF can be expressed as

$$
\left|\chi\left(t_{d}, f_{d}\right)\right| \triangleq\left|\int_{-\infty}^{\infty} u(t) u^{*}\left(t+t_{d}\right) \exp \left\{j 2 \pi f_{d} t\right\} d t\right|
$$

where $(\cdot)^{*}$ denotes the conjugate operator, $u(t)$ is the radar waveform, $t_{d}$ and $f_{d}$ are the mismatch in time delay and Doppler frequency, respectively. The ambiguity function plays a great role in determining the $t_{d}$ and $f_{d}$ resolutions of radar. The value of this function is maximum at $\left(t_{d}, f_{d}\right)=(0,0)$, and it represents the matched filter output without any mismatch. Hence, the narrower the AF around origin, the better the $t_{d}$ and $f_{d}$ resolutions. Therefore, the ideal AF would be an impulse at the origin, however, there is no waveform promising such kind of an AF.

\section{Frequency Diverse Array And Phased Array Radar Ambiguity Function}

First, we will analyzed FDA signal model and its ambiguity function derivation and then followed by phased array radar. FDA elements can be either excited by the same waveform or different waveforms. Consider $N$ elements of FDA radar signal model. Assume the $n$th element transmitted signal is given by

$$
\psi_{n}(t)=\exp \left(j 2 \pi z_{n, 1} t\right) s(t)
$$

where $z_{n, 1}$ denotes the $n$th transmit signal pulse carrier frequency and $s(t)$ can be expressed as

$$
s(t) \triangleq \begin{cases}1, & t \in\left[0, T_{\psi}\right] \\ 0, & \text { otherwise }\end{cases}
$$

with $T_{\psi}$ denotes the pulse duration. The $n$th FDA element emits the signal $\psi_{n}(t)$ with a frequency increment $\Delta f_{n}$. The signals radiated by the $n$th element can be expressed as

$$
\begin{aligned}
s_{n}(t) & =\sqrt{N} \psi_{n}(t) \exp \left(-j 2 \pi f_{n} t\right) w_{n}^{*} \\
& =\sqrt{N} u_{n}(t) w_{n}^{*}
\end{aligned}
$$

where $f_{n}=f_{0}+\Delta f_{n}$, with $f_{0}$ denotes the FDA radar carrier frequency, $w_{n}$ being weights and $u_{n}(t)$ defined in (5)

$$
u_{n}(t)=\exp \left(-j 2 \pi\left(f_{0}-z_{n, 1}+\Delta f_{n}\right) t\right) s(t)
$$

By following [16], the transmitted signal propagating along $(\theta, r)$ in the far-field is given by

$$
S_{n}(t, \theta, r)=\sqrt{N} \sum_{n=1}^{N} w_{n}^{*} a_{n}(t, \theta, r) u_{n}(t)
$$

and $a_{n}(t, \theta, r)$ can be written as [22]

$$
a_{n}(t, \theta, r)=e^{\left\{j 2 \pi\left(\Delta f_{n} t-\frac{\Delta f_{n} r}{c}+n \frac{d f_{0} \sin \theta}{c}\right)\right\}}
$$

Without loss of validity, a single-antenna is employed in the receiver. Assumed the round-trip time delay to a target in the angle $\theta$ and range $r$ is $t_{d}$ and $w_{n}=1$ is used. The received signal is written as

$\hat{r}_{s}\left(t, t_{d}, f_{d}, \theta, r\right)=\sqrt{N} \sum_{n=1}^{N} a_{n}(t, \theta, r) u_{n}\left(t-t_{d}\right) \exp \left(j 2 \pi f_{d} t\right)$

where $f_{d}$ denotes the Doppler frequency. When the received signal is matched with the assumed parameters $\left(t_{d}{ }^{\prime}, f_{d}{ }^{\prime}, \theta^{\prime}, r^{\prime}\right)$, the output of the matched filter is used to define FDA radar ambiguity function as expressed in (9), where the cross-ambiguity function is

$$
\chi_{n, n^{\prime}}\left(t_{d}, f_{d}\right) \triangleq \int_{0}^{T_{\psi}} u_{n}(t) u_{n^{\prime}}{ }^{*}\left(t+t_{d}\right) \exp \left(j 2 \pi f_{d} t\right) d t
$$

The following summarized FDA radar characteristics:

(1) If the frequency increment $\Delta f_{n}$ is fixed, the beam direction is a function of range $r$ dependent.

(2) If the range $r$ is fixed, the beam direction is a function of $\Delta f_{n}$ dependent.

(3) If the frequency increment $\Delta f_{n}$ is not employed $\left(\Delta f_{n}=0\right)$, the corresponding FDA simplified to phased array radar.

(4) As the frequency increment $\Delta f_{n}$ influences the beamwidth, a higher resolution may be achieved for the FDA as compared to the phased array radar.

By using the expression of the FDA radar ambiguity function derived in (9), the phased array ambiguity function can also be derived in a similar manner as in (11)

\section{Simulation Results And Discus- sions}

For the simulations, we consider a uniform linear FDA and phased array antenna with 16 elements spaced by $d$. The radar operational frequency is $10 \mathrm{GHz}$, frequency increment adopted is $30 \mathrm{kHz}, r=10 \mathrm{kHz}$ and $\theta=\frac{\pi}{3}$.

Figure 1(a) shows phased array radar ambiguity function when $\theta=\frac{\pi}{3}$ is adopted and Figure 1(b) depicts FDA radar ambiguity function when $r=10 \mathrm{kHz}$ and $\theta=\frac{\pi}{3}$ is also adopted. It can be seen in Figure 1(a) that the phasedarray radar has range-Doppler coupled and defocused ambiguity function. This implies that the phased-array radar cannot effectively distinguish range-angle dependent targets, while in Figure 1(b), FDA radar ambiguity function accumulates into a spike with relatively low sidelobe peak. It is evident from Figure 1(b) that, FDA radar can effectively distinguish range-angle dependent targets.

Figure (2a) and 2(b) illustrates the ambiguity functions in time delay and Doppler frequency dimensions. It can be observed in Figure 2(b) that by employment of frequency increment in FDA radar system produces a more focused peak which is useful in target localization than the phased array radar in Figure 2(a).

Figure 3(a) and 3(b) shows the comparisons of ambiguity function versus time delay of phased-array and FDA 


$$
\begin{aligned}
\chi\left(t_{d}, f_{d}, \theta, r, t_{d}{ }^{\prime}, f_{d}^{\prime}, \theta^{\prime}, r^{\prime}\right) & =\int_{-\infty}^{\infty} \hat{r}_{s}^{H}\left(t, t_{d^{\prime}}, f_{d^{\prime}}, \theta^{\prime}, r^{\prime}\right) \hat{r}_{s}\left(t, t_{d}, f_{d}, \theta, r\right) d t \\
& =N \cdot \sum_{n=1}^{N} \sum_{n^{\prime}=1}^{N}\left\{\exp \left\{j 2 \pi\left(\begin{array}{c}
T_{0}\left(\Delta f_{n}-\Delta f_{n^{\prime}}\right)-\frac{\left(\Delta f_{n}-\Delta f_{n^{\prime}}\right)}{f_{0} d\left((n-1) \sin \theta-\left(n^{\prime}-1\right) \sin \theta^{\prime}\right)} \\
+\frac{f^{\prime}}{c}
\end{array}\right)\right\} \chi_{n, n^{\prime}}\left(t_{d}, f_{d}\right)\right\}
\end{aligned}
$$

$$
\begin{aligned}
\chi\left(t_{d}, f_{d}, \theta, t_{d}^{\prime}, f_{d}^{\prime}, \theta^{\prime}\right) & =\int_{-\infty}^{\infty} \hat{r}_{s}^{H}\left(t_{d}^{\prime}, f_{d}^{\prime}, \theta^{\prime}\right) \hat{r}_{s}\left(t_{d}, f_{d}, \theta,\right) d t \\
& =N \cdot \sum_{n=1}^{N} \sum_{n^{\prime}=1}^{N}\left\{\exp \left\{j 2 \pi\left(\frac{f_{0} d\left((n-1) \sin \theta-\left(n^{\prime}-1\right) \sin \theta^{\prime}\right)}{c}\right)\right\} \chi_{n, n^{\prime}}\left(t_{d}, f_{d}\right)\right\}
\end{aligned}
$$

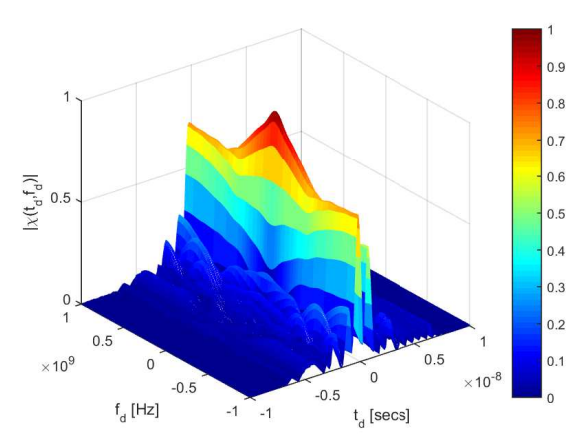

(a)

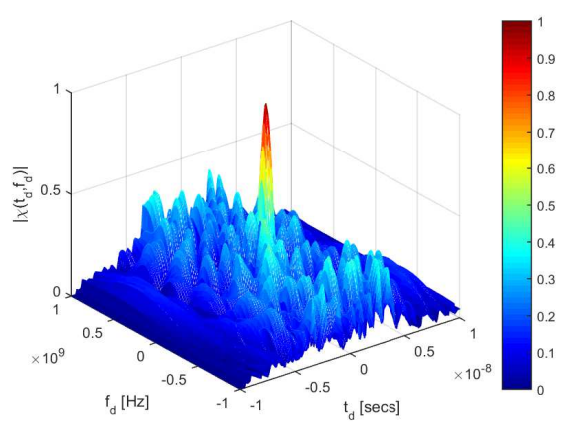

(b)

Figure 1: Comparisons of 3-D ambiguity functions: a) Phased-array radar, b) FDA radar.

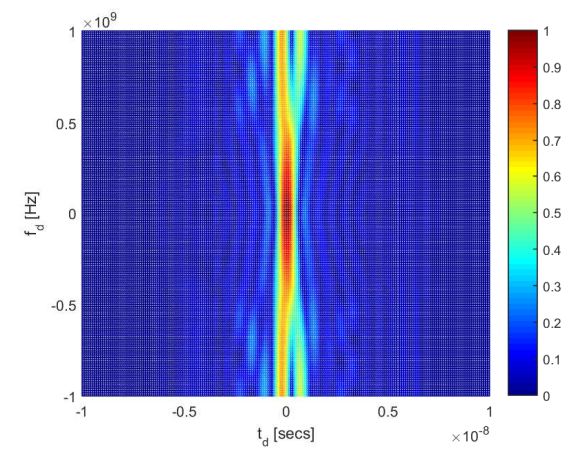

(a)

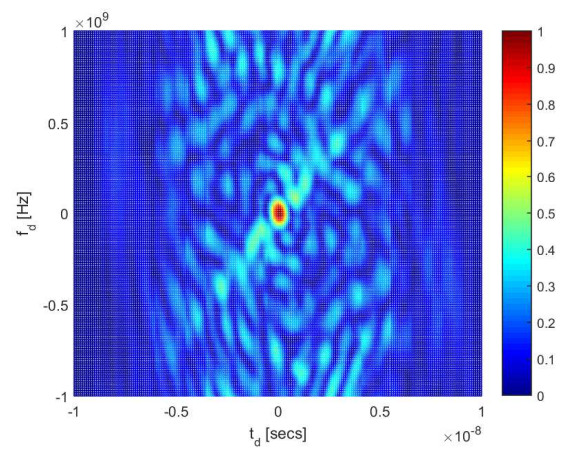

(b)

Figure 2: Comparisons of ambiguity functions: a) Phased-array radar, b) FDA radar.

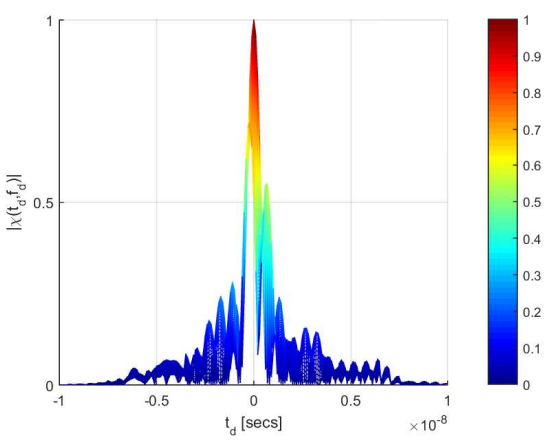

(a)

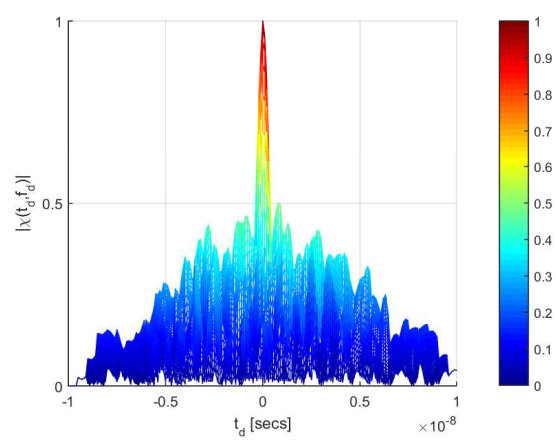

(b)

Figure 3: Comparisons of ambiguity function versus time delay: a) Phased-array radar, b) FDA radar. 


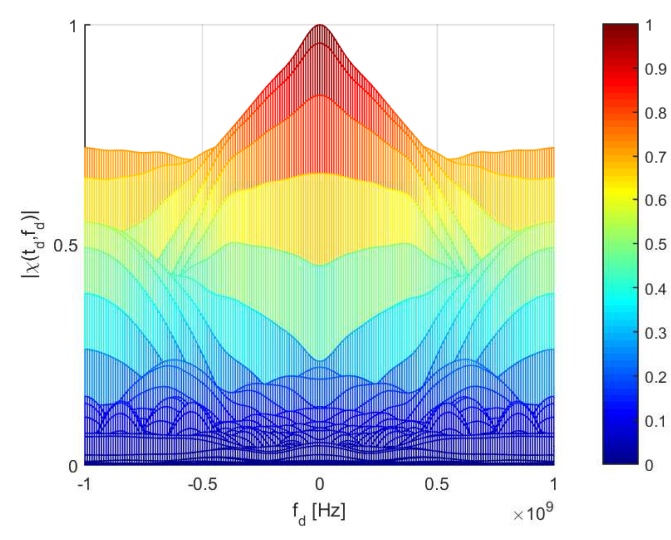

(a)

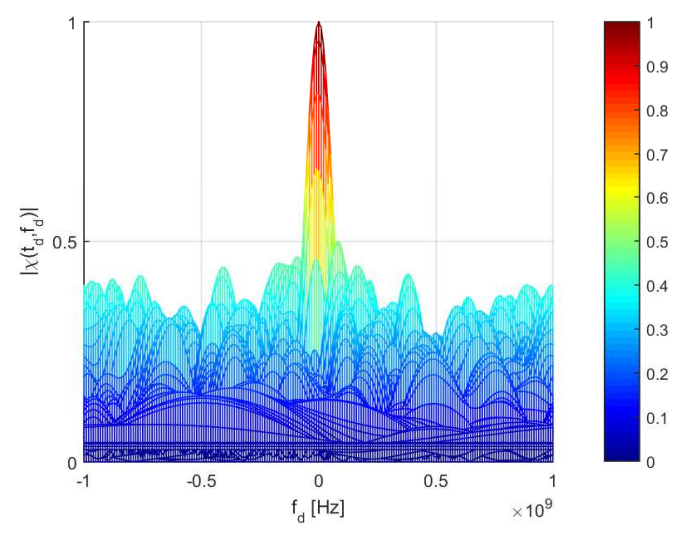

(b)

Figure 4: Comparisons of ambiguity function versus Doppler frequency: a) Phased-array radar, b) FDA radar.

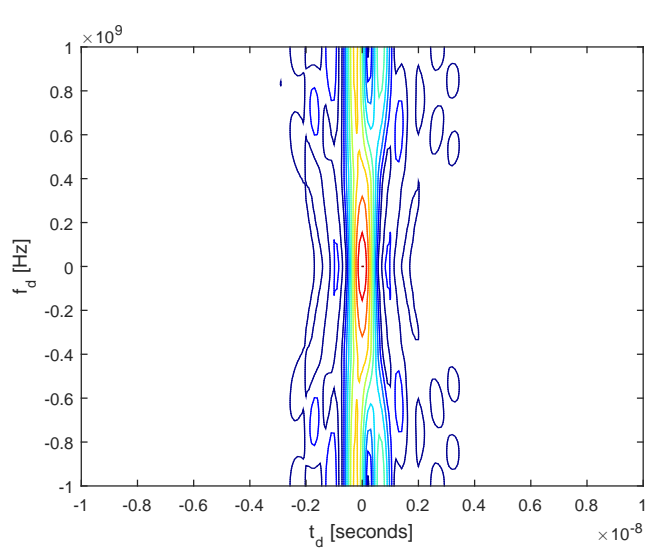

(a)

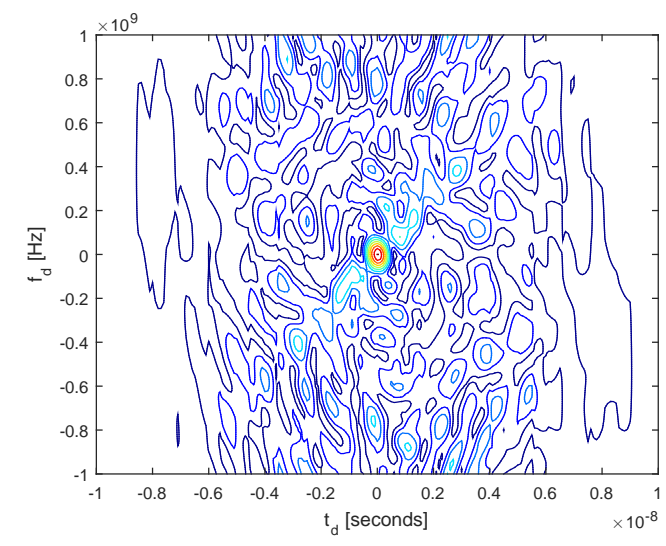

(b)

Figure 5: Comparisons of contour plot: a) Phased-array radar, b) FDA radar.

radar, respectively. It can be noticed in Figure 3(a) that the phased-array radar produces narrow beamwidth with maximum sidelobe peak ratio of $0.5510 \mathrm{~dB}$. On the other hand, the FDA radar in Figure 3(b) also achieves a narrow beamwidth with maximum sidelobe peak ratio of 0.5003 dB. Hence, FDA radar outperforms phased array in terms of sidelobe peak ratio.

Figure 4(a) and 4(b) compares the ambiguity function versus Doppler frequency of phased-array and FDA radar, respectively. It can be observed in Figure 4(a) that the phased-array radar produces poor Doppler frequency resolution which is coupled. In contrast, the FDA radar in Figure 4(b) produces a narrow beamwidth Doppler frequency resolution with maximum sidelobe peak ratio of $0.5003 \mathrm{~dB}$. Therefore, FDA radar can estimates Doppler frequency effectively than phased array radar.

Figure 5(a) and 5(b) examines the contour plots of phased-array and FDA radar systems, respectively. It can be seen in Figure 5(b) that the FDA radar contour plot produces a narrow resolution in time delay and Doppler frequency dimensions than the phased array radar in Figure 5(a). It is also evident from Figure 5(b) that the fuzzy area will reduce with increase in $N$, which would also finally affect the time delay and Doppler frequency resolutions of the system.
Finally, an effective metric used to evaluate the ambiguity function is the empirical cumulative distribution function (ECDF), which represents the percentage of samples of $\left|\chi\left(t_{d}, f_{d}\right)\right|$ lower than a given magnitude. Figure 6 illustrates the ECDF curve to compare both radar systems. The ECDF performance means that the FDA radar yields smaller sidelobe energy than the phased array radar.

\section{Conclusion}

In this paper, FDA radar and Phased-array radar ambiguity functions has been formulated. Simulation results shows that the phased-array radar has range-Doppler coupled and defocused ambiguity functions. This implies that the phased-array radar cannot effectively distinguish rangeangle dependent targets. In contrast, the FDA radar ambiguity obtained lower maximum sidelobe level without degrading the mainlobe performance and can effectively distinguish range-angle dependent targets. In addition, the performance analysis has also been done in terms of empirical cumulative distribution function (ECDF) which proved that FDA radar ambiguity function outperforms the phased array radar ambiguity function. Furthermore, FDA radar ambiguity function produces narrow resolution in time delay and 


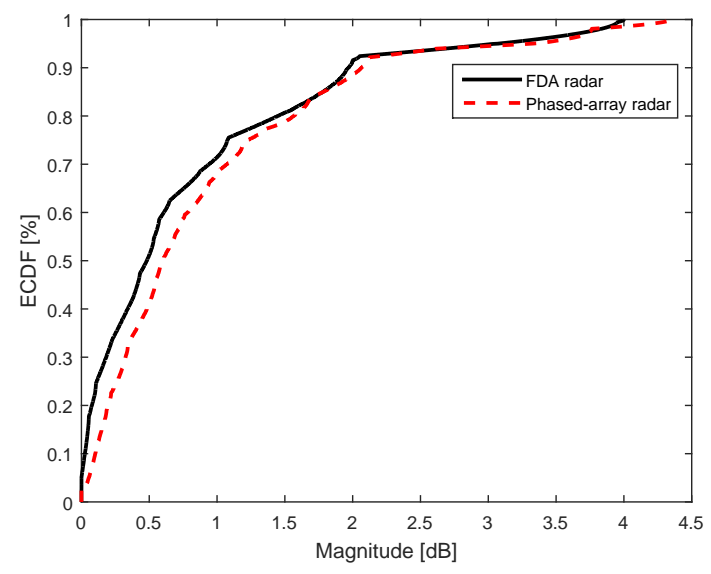

Figure 6: Comparisons of ECDF performances.

Doppler frequency dimensions than its counterpart phasedarray radar.

Conflict of Interest The author declare no conflict of interest.

Acknowledgment The author would like to thank Respected Professor Wen-Qin Wang (Senior Member - IEEE) of UESTC for his great contributions, support and encouragement.

\section{References}

[1] K. T. Wong, Y. I. Wu, Y. S. Hsu, and Y. Song. "A lower bound of DOA estimates by an array randomly subject to sensor-breakdown,'IEEE Sensors J., vol. 12, no. 5, pp. 911-913, May 2012.

[2] N. I. Giannoccaro and L. Spedicato. "A new strategy for spatial reconstruction of orthogonal planes using a rotating array of ultrasonic sensors,'IEEE Sensors J., vol. 12, no. 5, pp. 1307-1316, May 2012.

[3] S. Yong and J. T. Bernhard. "A pattern reconfigurable null scanning antenna,'IEEE Trans. Antennas Propag., vol. 60, no. 10, pp. 4538-4544, Oct. 2012.

[4] J. J. Adams and J. T. Bernhard. "A modal approach to tuning and bandwidth enhancement of an electrically small antenna,'IEEE Trans. Antennas Propag., vol. 59, no. 4, pp. 1085-1092, Apr. 2011.

[5] W.-Q. Wang. "Mitigating range ambiguities in high PRF SAR with OFDM waveform diversity,'IEEE Geosci. Remote Sens. Letter, vol. 10, no. 1, pp. 101105, Jan. 2013.

[6] P. Antonik, M. C. Wicks, H. D. Griffiths, and C. J. Baker. "Frequency diverse array radars,"in Proc. IEEE Radar Conf., Verona, Italy, Apr. 2006, pp. 215-217.

[7] P. Antonik, M. C. Wicks, H. D. Griffiths, and C. J. Baker. "Multi-mission multi-mode waveform diversity,"in Proc. IEEE Radar Conf., Verona, Italy, Apr. 2006, pp. 580-582.
[8] P. Antonik, M. C. Wicks. "Method and apparatus for simultaneous synthetic aperture and moving target indication,’U.S. Patent 0129584, Jun. 5, 2008.

[9] P. Antonik. "An investigation of a frequency diverse array,’Ph.D. dissertation, Dept. Electron. Electr. Eng., Univ. College London, London, U.K., 2009.

[10] A. Aytun. "Frequency diverse array radar,"M.S. thesis, Dept. Comput. Sci., Naval Postgraduate School, Monterey, CA, USA, 2010.

[11] S. Brady. "Frequency diverse array radar: Signal characterization and measurement accuracy,"M.S. thesis, Dept. Math., Air Force Inst. Technol., Patterson AFB, OH, USA, 2010.

[12] P. Antonik, M. C. Wicks, H. D. Griffiths, and C. J. Baker. "Range dependent beamforming using element level waveform diversity,"in Proceedings of the International Waveform Diversity and Design Conference,Las Vegas, USA, January 2006, pp. 1-4.

[13] S. Mustafa, D. Simsek, and H. A. E. Taylan. "Frequency diverse array antenna with periodic time modulated pattern in range and angle,"in Proceedings of the IEEE Radar Conference, Boston, April 2007, pp. 427-430.

[14] S. Huang, K. F. Tong, and C. J. Baker. "Frequency diverse array: Simulation and design,"in Proceedings of the LAPS Antennas and Propagation Conference, Loughborough, UK, May 2009, pp. 253-256.

[15] J. Farooq. "Frequency diversity for improving synthetic aperture radar imaging,"Ph.D. dissertation, Air Force Institute of Technology, 2009.

[16] W.-Q. Wang and H. C. So. "Transmit subaperturing for range and angle estimation in frequency diverse array radar,'IEEE Transactions on Signal Processing, vol. 62, no. 8, pp. 2000-2011, April 2014.

[17] Y. B. Wang, W.-Q. Wang, and H. Chen. "Linear frequency diverse array manifold geometry and ambiguity analysis,'IEEE Sensors Journal, vol. 15, no. 2, pp. 984-993, February 2015. 
[18] N. Levanon and E. Mozeson. "Radar Signals,"New York, NY, USA: Wiley-IEEE Press, 2004.

[19] N. Levanon and E. Mozeson. "Removing autocorrelation sidelobes by overlaying orthogonal coding on any train of identical pulses,'IEEE Trans., Aerospace and Electronics Systems, vol. 39, no. 2, April 2003.

[20] Dennis W. Ricker. "Echo signal processing,"The Springer International Series in Engineering and Computer Science, vol.725, 2003.
[21] Lu Zhang, Bo Yang, and Mao Kang Luo. "Joint delay and Doppler shift estimation for multiple targets using exponential ambiguity function,'IEEE Transactions on Signal Processing, DOI 10.1109/TSP.2017.2655494, pp. 1-13, 2017.

[22] W.-Q. Wang, H. C. So, and H. Z. Shao. "Nonuniform frequency diverse array for range-angle imaging of targets,'IEEE Sensors Journal, vol. 14, no. 8, pp. 2469-2476, August 2014. 